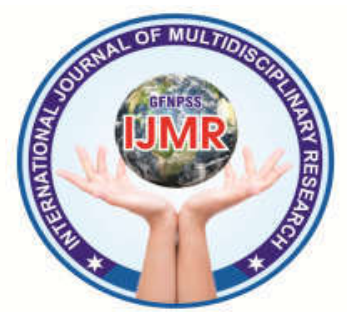

\title{
ANTIBIOTIC PROPHYLAXIS IN THIRD MOLAR SURGERY
}

\section{DR. AMIT K SHARMA ${ }^{1}$ DR. SANCHAYNEE K. PATHAK ${ }^{2}$ DR. ABHINAV NEOG $^{3}$ DR. HARSHESH K. PATEL ${ }^{4}$}

${ }^{1}$ MDS (Oral \& Maxillofacial Surgery), Professor \&HOD Department of Oral and Maxillofacial Surgery, Daswani Dental College and Research Centre Kota, Rajasthan.

${ }^{2}$ MDS (Oral and Maxillofacial Surgery), Senior Lecturer, Department of Oral and Maxillofacial Surgery, Daswani Dental College and Research Centre Kota, Rajasthan.

${ }^{3}$ MDS (Oral and Maxillofacial Surgery), Project Scientific Medical Officer B, NCDIR-

ICMR Dr. B. Borooah Cancer Institute, Guwahati, Assam

${ }^{4}$ Post Graduate Student, Department of Oral and Maxillofacial Surgery, Daswani Dental College and Research Centre Kota, Rajasthan.

Corresponding Email: bala.dr0359@gmail.com,sanchupathak123@icloud.com, abhinav.neog@gmail.com, nats3014@gmail.com

\section{ABSTRACT}

Evaluate: The aim of this study is to evaluated the influence of antibiotic prophylaxis on third molar removal in young patients.

Material and Methods: 100 patients (50 males and 50 females, age range 18-40 years) requiring lower $3^{\text {rd }}$ molar extractions were selected and divided randomly in two groups; A (test group) and B (control group). Group A received $2 \mathrm{~g}$ amoxicillin tablet an hour prior to surgery and Group B received no antibiotic therapy.

Results: In group A there was a significant reduction of postoperative pain experienced in post-operative 7 days as compared to group B. Swelling was presents in all patient of group B and in only 2 patients of group A. Sequalae of wound infection reported in 4 patients of group B and 1 patient in group A. Fever was present in 2 patients of group B and 1 patient in group A.

Conclusions: A statistically significant difference was found between patients receiving preoperative amoxicillin with respect to postoperative pain, fever, and wound infection.

Keywords: Antibiotics, Prophylaxis, Mandibular third molar, Amoxicillin, Trans-alveolar Extraction. 


\section{INTRODUCTION}

In immunocompromised patients or in patients affected by diabetes or cardiovascular disease, antibiotic prophylaxis is a well-established routine therapy before surgery ${ }^{1}$.It has been reported in the literature that in medically healthy patients, there is no indication for antibiotic prophylaxis ${ }^{1}$; however, some recent studies reported some positive effects regarding reduction of pain and wound infection after preoperative and postoperative antibiotic therapy ${ }^{2}$.

The healing process after the extraction of an impacted third molar depends on different variables such as surgeon experience, patient age, presence of periodontal pathology, and necessity for bone removal and tooth sectioning of the third molar if deeply impacted ${ }^{3-7}$. To evaluate this claim of authors, we decided to test the principles of antibiotic prophylaxis in the removal of third molar in young patients who all had mesioangular impaction to obtain better standardization of the surgical protocol.

\section{MATERIALS AND METHODS}

The study included collecting data from 100 (50 males and 50 females, mean age 26)patients coming to the department of Oral and MaxillofacialSurgery during the period from March 1, 2018 to February 25, 2019 for extraction of their lower third molar. The ethical approval was obtained from the local ethical committee at Jaipur Dental College, Jaipur, Rajasthan. Variables liked to angle of mandible, root shape; were standardized as mesioangular impaction with no dilacerations. To keep the study unbiased in regards of surgical expertise; only one surgeon performed all the cases.

\section{Inclusion Criteria were:}

(1) Patients in the range of $18-40$ years of age.

(2) Patients with partially bony mesioangular impacted mandibular third molar without pericoronitis, periapical and periodontal diseases.

\section{Exclusion Criteria were:}

(1) Immunocompromised patients.

(2) Patients having local pathology such as a cyst or tumor associated with impacted tooth,

(3) Patients with irradiated maxillofacial region,

(4) Mentally challenged patients.

(5) Patients in whom the surgical phase extended for more than $1 \mathrm{~h}$.

\section{Methodology}

Patients were randomized into two groups; A (test group) and B (control 


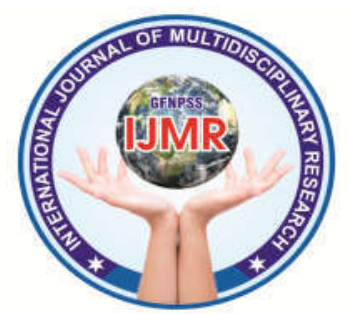

group).Group A received $2 \mathrm{~g}$ amoxicillin tablet an hour prior to surgery

All the procedures were performed under local anesthesia by the same technique. Complete aseptic precautions were used. Constant copious irrigation with saline was used during ostectomy and odontomy using micromotor with straight handpiece. Sectioning of the tooth was done whenever indicated. Primary closure was accomplished with 3-0 Silk sutures.

All the patients were given the following set of instructions:

(1) Apply ice pack for the first $48 \mathrm{~h}$ after surgery $-10 \mathrm{~min}$ per every $2 \mathrm{~h}$ in the first $24 \mathrm{~h}$, and then twice a day for the next $24 \mathrm{~h}$

(2) Take cold soft, liquid/semi solid diet,

(3) Take the prescribed analgesics

(4) Patients were asked to report on $2^{\text {nd }}$, $3^{\text {rd }}, 5^{\text {th }}$ and $7^{\text {th }}$ post - operative days to check the following parameters and for the wound infection analysis was done after $1^{\text {st }}, 2^{\text {nd }}$ and $3^{\text {rd }}$ week.

Evaluation of Pain was by Visual Analogue Scale [8]

Score 0 - No pain.

Score 1-Slight pain; If patient is distracted he/she does not feel pain.

Score 2-Mild pain; The patient feels pain even if concentrating on some activity.
Score 3-Severe pain; The patient is very disturbed but nevertheless can continue with the normal activities.

Score 4-Very severe pain; The patient is forced to abandon normal activities.

Score 5-Extremely severe pain.

\section{Analgesic Consumption}

Analgesics prescribed for the patients who reported with severe pain after postoperative week.

\section{Variables Score}

No - 0

Yes - 1

\section{Wound Infection}

Patients were evaluated for wound infection at each visit, halitosis, pain, clinical signs of clot less socket with necrotic bone were used as diagnostic criteria.

\section{Variables Score}

None - 0

Yes -1

\section{Evaluation of Facial Measurement}

The patients who reported swelling were rated on a scale [8] from 1 to 3 as: 1 -swelling visible but not sufficient to alter the patient's face, 2-swelling sufficient to alter the patient's face but not enough to limit mouth opening, and 


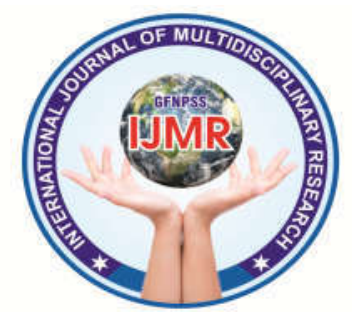

3-swelling associated with trismus that

Wound infection was reported in 4 patients limited mouth opening

Temperature $\left(>38^{\circ} \mathrm{C}\right)$

Patient's axillary temperature was of group B and 1 patient in group A. In group B, 1 case with wound infection recorded pre-operatively and on every visit.

\section{Variables Score}

No - 0

Yes $\left(>38^{\circ} \mathrm{C}\right)-1$

\section{RESULTS}

In this study, Sever Pain was present in 8 extractions in the group $\mathrm{B}$ and in 1 extraction in the group A. In the group B pain persisted for the first 3 to 5 days in 6 patients and entire post extraction week in 2 patients and in all other patients' pain was present for 1 or 2 days. In the group A only 1 patient reported of pain in first 3 days. Regarding pain, the difference between the $\mathrm{A}$ and $\mathrm{B}$ groups was statistically significant (Figure 1).

In terms of the use of analgesics, the mean consumption of analgesics in the postoperative week for each patient in group B was 9 whereas; in the group A it was 3 ; the mean postoperative consumption of analgesics was higher in the group $\mathrm{B}$ when compared with the group A, and this difference was statistically significant (Figure 2).

became evident 1 week after the extraction, 1 case 15 days after extraction, and 2 more cases 3 weeks after extraction. In the group A only 1 patient had wound infection who reported 3 weeks after the extraction (Figure 3).

Postoperative swelling was present in 48 of 50 extractions performed with antibiotic prophylaxis (group A) and in all 50 extractions of group B. Swelling was present in the first 3 days after extraction, increasing especially on the $2^{\text {nd }}$ and $3^{\text {rd }}$ day. In the group $\mathrm{B}$, the swelling was scored as 3 in 2 case, as 2 in 31 cases, and as 1 in 17 cases. In the group $A$ it was scored as 2 in 30 cases and as 1 in 18 cases, and it was absent in 2 cases.

We decided to compare the total number of cases with a score of 2 and a score of 3 in both the groups. In this manner we found that swelling was present in 33 cases in the group B and 30 cases (all with a score of 2) in the group A; this difference was not statistically significant.

Fever was present in 2 patients in the group B and in 1 patient in the group A. This difference was not statistically significant; thus, we have not produced a 


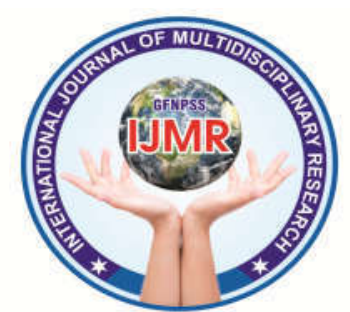

graphical representation for fever as well for swelling.

\section{DISCUSSION}

Prophylactic antibiotics have been used to decrease the risk of surgical wound infection, but its role in preventing infection in all situations is situation is controversial. In his landmark animal study that defined the scientific basis of prophylaxis, Burke [9] has clearly stated that the use of post-operative antibiotics alone actually actually violates the basic tents of prophylaxis because there is no antibiotics either in the systemic circulation or at the site of surgery when the microorganisms invade the wound.

The meta-analysis of randomized controlled clinical trials performed by Ren and Malmstrom [10]on the effectiveness of antibiotic prophylaxis in third molar surgery reported an effect of antibiotic therapy in reducing alveolar osteitis and wound infection after third molar extraction. The study of Lacasa et al [2] reported the same effect on wound infection and in pain after postoperative antibiotic therapy. In our present study, antibiotic prophylaxis was effective in preventing postoperative pain and wound infection after extraction of third molars. Moreover, the analgesic consumption was minimal in the test group, and these results are in accord with those of Lacasa et al [2]. Regarding the type of chemical principle tested, amoxicillin was one of the most tested antibiotics in previous studies, and it could be less efficacious if compared with other more recently introduced antibiotics, but it has fewer collateral effects. Amoxicillin, as reported in British National Formulary 53 2007, is a firstchoice antibiotic for a short-term treatment of infections in the mouth [11].A single high dose of antibiotic taken 1 hour before the contaminated surgery, which we consider the removal of impacted teeth to, conforms with the cited indications and reduces the chance for any possible abuse that is responsible for antibiotic resistance. The purpose of this study was to evaluate, this type of antibiotic prophylaxis could result in a better course and better wound healing. We believe that patients undergoing contaminated surgery could take amoxicillin only as prophylaxis and could be given more effective antibiotics in the postoperative period if serious complications occur. We applied for amoxicillin to verify which protocol is more effective.

Another interesting fact we encounter was that female patients had less pain than 


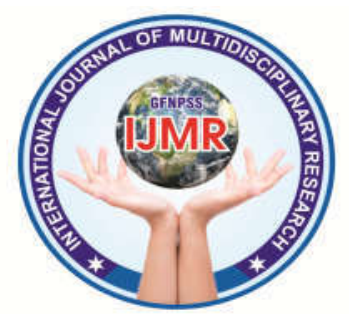

male patients, but this difference was not

ACKNOWLEDGEMENTS AND

statistically significant, probably because DISCLOURE STATEMENTS

the number of patients was low. This could

The authors declare no conflict of be related to the deleterious habits which are probably found lesser in females than males. But it cannot be validated unless a proper data has been procured.

\section{CONCLUSION}

Lower third molar removal is a common surgical procedure performed in dentistry that often results in pain, swelling, trismus, bleeding, nerve dysfunction, and postoperative infection. In addition, antibiotic therapy after impacted lower third molar removal is common and, until recently, universally accepted. The current study suggests the benefits of routine prophylactic antibiotic therapy which does not appear to overcome the risk of undesirable outcomes after third molar removal. In our opinion and the findings of this study are important because for this specific target group of patients (between aged 18-40 years), the antibiotic prophylaxis resulted in relative decrease of postoperative pain and consistent reduction of analgesic consumption and is therefore recommended to reduce thepsychological impact of this type of surgery.

interest. Also, this study did not receive any specific grant from funding agencies in public, commercial, or non-profit sectors.

\section{REFERENCES}

1. Zeitler DL. Prophylactic antibiotics for third molar surgery.J Oral MaxillofacSurg. 1995 Jan;53(1):6164. [Medline: 7799123]

2. Lacasa JM, Jimenez JA, Ferras V,Bossom M, Sola-Morales O, GarciaRey C, Aguilar L, Garau J. Prophylaxis versus preemptive treatment for infective and inflammatory complications of surgical third molar removal: A randomized, doubleblind, placebo-controlled, clinical trial with sustained release amoxicillin/clavulanic acid (1000/62.5 mg).IntJ OralMaxillofac Surg. 2007 Apr;36(4):321-327. [Medline: 172295 48]doi:10.10/j.ijo.2006.11.007]

3. Monaco G, Staffolani C, Gatto MR, Checchi L. Antibiotic therapy in impacted third molar surgery.EurJ Oral Sci. 1999 Dec; 107(6):437-441. [Med line: 10625102$]$ 


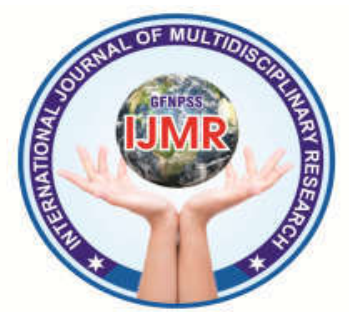

4. Capuzzi P, Montebugnoli L, Vaccaro MA. Extraction of impacted third molars. A longitudinal prospective study on factors that affect postoperative recovery.Oral Surg Oral Med Oral Pathol. 1994 Apr;77(4):341-343. [Medline: 8015796]

5. Haug RH, Perrott DH, Gonzales ML, Talwar RM. The American Association of Oral and Maxillofacial Surgeons Age-Related Third Molar Study. JOral MaxillofacSurg. 2005 Aug;63(8):1106-1114. [Medline: 16094577] [doi: 10.1016/j.joms.200 5 $.04 .022]$

6. Piecuch JF, Arzadon J, Lieblich SE: Prophylactic antibiotics for third molar Surgery: A supportive opinion. J OralMaxillofacSurg. 1995 Jan;53(1):53-60. [Medline: 7799122]

7. Poeschl PW, Eckel D, Poeschl E. Postoperative prophylactic antibiotic treatment in third molar surgery-A necessity? J Oral MaxillofacSurg. 2004 Jan;62(1):3-8.Medline:14699540
8. Berge TI . Visual analogue scale of postoperative pain and swelling. Acta Odontol Scand. 1988 Aug;46(4):233240. [Medline: 3188849]

9. Burke JF. The effective period of preventive antibiotic action in experimental incisions and dermal lesions.Surgery. 1961 Jul;50:161-8. [Medline: 16722001]

10. Ren YF, Malmstrom HS: Effectiveness of antibiotic prophylaxisin third molar surgery: A meta-analysis of randomized controlledclinical trials. $\mathrm{J}$ Oral MaxillofacSurg. 2007 Oct;65(10):1909-1921. [Medline: 17884515] [doi: 1016/j.joms .2007.0 3.004]

11. Martin, Mehta, D.K. British National Formulary 53.BMJ Publishing Group, Royal Pharmaceutical Society Of Great Britain, London, UKMarch 2007.p. 278. 